\title{
Participación de los padres en el rendimiento académico de alumnos de primaria. Revisión de literatura
}

\author{
Parental Involvement in Academic Achievement of Elementary School Students. \\ A Literature Review
}

\author{
Participação dos pais no desempenho acadêmico dos alunos do ensino \\ fundamental. Revisão de literatura
}

\author{
Edwin Alexis Mayorquín Reyes \\ Universidad del Pacífico Norte, México \\ mareea_10@hotmail.com \\ https://orcid.org/0000-0002-7105-0051 \\ Aníbal Zaldívar Colado \\ Universidad Autónoma de Sinaloa, México \\ azaldivar@uas.edu.mx \\ https://orcid.org/0000-0002-6622-6630
}

\section{Resumen}

La educación es la base de la sociedad. Aun así, es frecuentemente confundida con adiestrar y adoctrinar; aunado a que sufre el desinterés de la familia, lo que facilita el fracaso escolar y provoca una creciente problemática en el rendimiento de los estudiantes desde hace años en México. Principalmente conformada por el triángulo maestro-alumno-padre de familia, requiere que estos elementos se desempeñen de manera adecuada para lograr el éxito académico. En el contexto mexicano, no obstante, la armonía entre las tres partes frecuentemente está corrompida y es ahí dónde surge el problema.

Este artículo revisa la literatura de investigación sobre la relación entre la participación de los padres y el rendimiento académico en estudiantes de educación primaria. Se trata de una revisión de 41 estudios publicados entre 2011 y 2019. Los resultados se clasifican en una tabla que incluye lugar y fecha de publicación, autor y principal contribución al área de estudio. Se discuten las coincidencias y divergencias de las publicaciones incluidas en este artículo; así como las tendencias futuras en esta línea de investigación. 


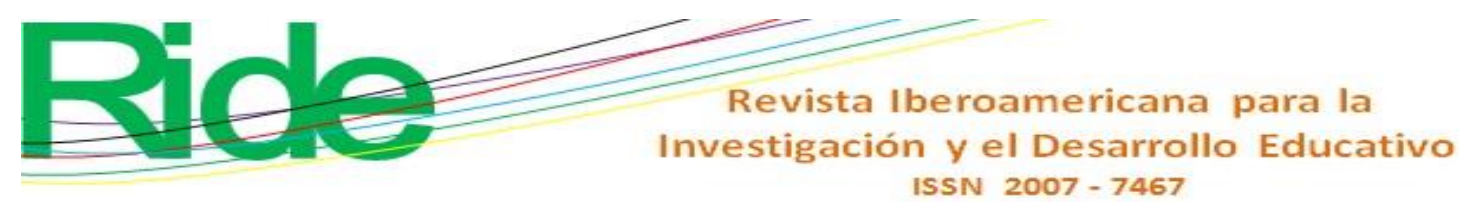

Palabras clave: análisis documental, educación básica, padres, rendimiento escolar.

\section{Abstract}

Even though education is the basis of society, it is often confused with training and indoctrination, and suffers the disinterest of the family, which facilitates school failure and causes a growing problem in the performance of students for years in our country. Mainly conformed by the teacherstudent-parent triangle, it requires that these elements perform adequately to achieve academic success. In Mexico, however, the harmony between the three parts is often corrupted, and that is where the problem arises.

This article reviews the research literature on the relationship between parental involvement and academic performance in primary education students. It is a review of 41 studies published between 2011 and 2019. The results are classified in a table that includes place and date of publication, author and main contribution to the study area. The coincidences and divergences of the authors included in this article are discussed; as well as future trends in this line of research.

Keywords: documentary analysis, basic education, parents, academic achievement.

\section{Resumo}

A educação é a base da sociedade. Mesmo assim, muitas vezes é confundido com treinamento e doutrinação; juntamente com a falta de interesse da família, o que facilita o fracasso escolar e causa um problema crescente no desempenho dos alunos durante anos no México. Principalmente moldado pelo triângulo professor-aluno-pai, requer que esses elementos tenham um desempenho adequado para alcançar o sucesso acadêmico. No contexto mexicano, no entanto, a harmonia entre as três partes é muitas vezes corrupta e é aí que o problema surge.

Este artigo revisa a literatura de pesquisa sobre a relação entre o envolvimento dos pais e o desempenho acadêmico em estudantes do ensino fundamental. Esta é uma revisão de 41 estudos publicados entre 2011 e 2019. Os resultados são classificados em uma tabela que inclui local e data de publicação, autor e contribuição principal para a área de estudo. As coincidências e divergências das publicações incluídas neste artigo são discutidas; bem como as tendências futuras nesta linha de pesquisa. 


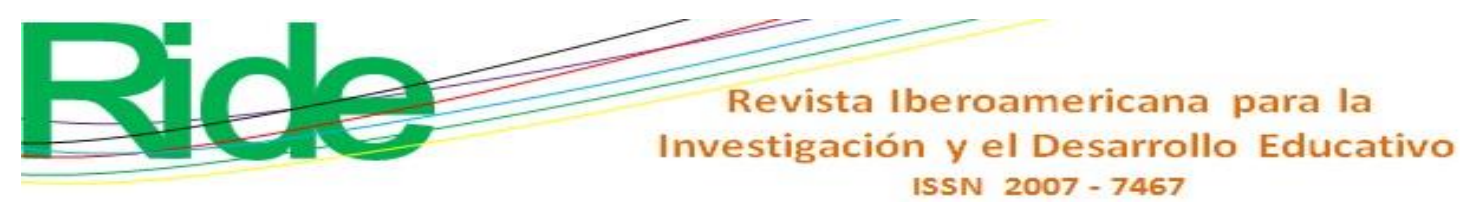

Palavras-chave: análise documental, educação básica, pais, desempenho escolar.

Fecha Recepción: Junio 2018

Fecha Aceptación: Noviembre 2018

\section{Introducción}

Un tema importante en la identificación de puntos de influencia para mejorar el rendimiento académico de los estudiantes en el nivel básico es determinar cómo y en qué medida la participación de sus padres afecta el rendimiento del alumno. Tal conocimiento podría contribuir en la reestructuración de las prácticas de crianza, así como en las políticas e intervenciones basadas en la escuela, en específico aquellas que implican trabajar con los padres. En efecto, una investigación de estas características ayudaría en el diseño e implementación de intervenciones que maximicen la participación de los padres, con el objetivo de tener un efecto positivo en el aprendizaje de los niños. En este trabajo se revisó la literatura actual sobre la influencia de los padres en el rendimiento de los alumnos. Por ahora tan solo se puede adelantar que las publicaciones son complejas y, en ocasiones, contradictorias. En síntesis, este documento pretende desentrañar el fenómeno al examinar de cerca resultados de investigación pertinentes sobre la relación de los padres y el rendimiento académico de sus hijos en la escuela primaria.

La idea de que la participación de los progenitores engendra los logros académicos de los estudiantes es intuitivamente atractiva. A tal grado de que la sociedad en general, y los educadores en particular, han considerado a aquellos, a los padres, como un ingrediente importante para el remedio de muchos males en la educación. Ya lo expusieron Shute, Hansen, Underwood y Razzouk (2011) y más recientemente Jhang y Lee (2018). Pero desde los 80 (Stevenson y Baker, 1987; Keith, Reimers, Fehrmann, Pottebaum, y Aubey, 1986) y principios de la década de 1990 (Epstein, 1991; Epstein y Dauber, 1991) se publicaron estudios que sugerían la importancia de la participación de los padres en la escuela. A mediados de 1990, los investigadores educativos defendieron activamente la influencia de los padres en la educación (Sui-Chu y Willms, 1996; Halle, Kurtz-Costes y Mahoney, 1997). Se promulgaron leyes como la Goals 2000: Educate America Act y la Ley de Educación Primaria y Secundaria, reautorizada en Estados Unidos, que ha convertido la participación de los padres en la educación de sus hijos en una prioridad nacional (Baker y Soden, 1998). Se ha alentado a las escuelas a reexaminar sus políticas y programas de 


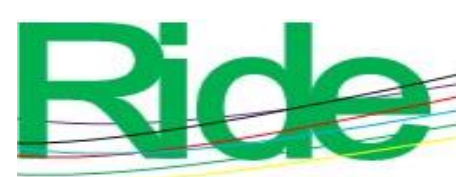

Revista Iberoamericana para la

Investigación y el Desarrollo Educativo

ISSN 2007 - 7467

participación de los padres y a utilizar enfoques innovadores para obtener financiamiento para la educación a través de programas federales y estatales — consejos de participación social en las escuelas.

Muchos profesionales e investigadores apoyan la dirección de políticas de aumento de injerencia de los padres en la vida académica de sus hijos, pero persiste la confusión en cuanto a una definición adecuada y las actividades, objetivos y resultados deseados. Poco se sabe sobre la incidencia de los padres en el rendimiento académico de sus hijos; los primeros estudios que sugieren su importancia, desafortunadamente, se tratan como definitivos, independientemente de la naturaleza equívoca de los datos, y se utilizan para respaldar la posición de que prácticamente cualquier tipo de influencia de los padres en la educación de sus hijos es importante. Esta influencia ha sido tratada durante mucho tiempo como una construcción unidimensional (Sui-Chu y Willms, 1996).

Recientemente se ha vivido dentro de la escuela primaria en México una problemática respecto al aprovechamiento escolar del alumnado (Instituto Nacional para la Evaluación de la Educación [INEE], 2018; Backoff, 2016). No es un fenómeno nuevo, sino que la calidad educativa viene deteriorándose desde hace una década o más (Gómez, 2017). Lo ideal sería contar con el interés, apoyo, responsabilidad y motivación de los padres de familia por la capacitación de sus hijos. Que ellos fueran los primeros en percatarse de los obstáculos en el aprendizaje que tienen sus hijos, así como en la formación como individuo (personalidad); y además, llevaran la batuta del accionar para superar trabas, o al menos intentarlo.

Esta investigación tiene como finalidad el análisis de una parte de la literatura sobre las características del apoyo presentado por los padres de familia y su relación con el aprovechamiento escolar de los estudiantes, con el objetivo de ayudar a la mejora de las prácticas docentes y sus relaciones con el entorno inmediato de la institución educativa; además de ayudar a comprender la realidad que se vive en la relación padre-hijo dentro de la educación primaria.

El resto del trabajo se estructura de la siguiente manera: en la segunda sección se detalla la metodología para realizar la propuesta de clasificación de los trabajos analizados en esta investigación, considerando a los autores que abordan el tema y aportando información útil tanto para apoyar este estudio como para contrastar la información obtenida. Enseguida se exponen los resultados en formato de tabla, donde se clasifican las publicaciones analizadas y con base en la 


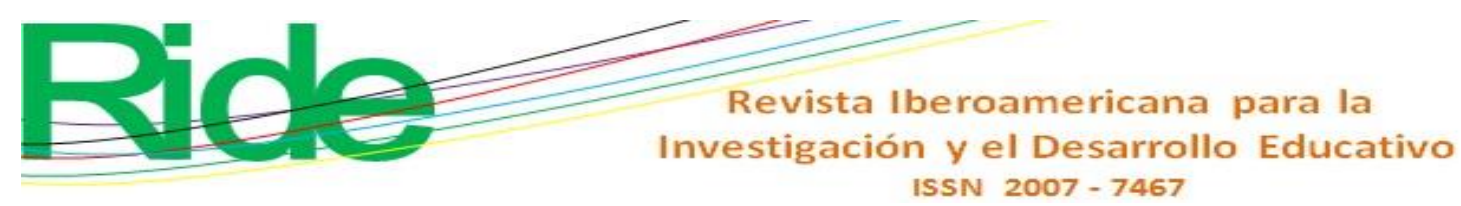

propuesta de la segunda sección. Después se mencionan las tendencias futuras de esta línea de investigación. Finalmente, se discuten las conclusiones de este trabajo.

\section{Involucramiento de los padres en el rendimiento académico}

Una gran cantidad de estudios analizan aspectos relacionados al involucramiento de los padres en el rendimiento académico de sus hijos, pero son pocos los que coinciden al correlacionar estas variables. Estas diferencias conducen a hallazgos contradictorios o evitan la evolución en el conocimiento sobre el área de estudio. Al analizar las publicaciones relacionadas, se detectaron al menos dos categorías: 1) intervención en el hogar (se refiere a lo que hacen los padres en el hogar para promover el aprendizaje de sus hijos) y 2) intervención en la escuela (actividades de participación de los padres en la escuela); ningún autor menciona la comunicación a través de Internet, el involucramiento de los padres de familia en el rendimiento de sus hijos en nivel primaria. Enseguida se enlistan los rubros más importantes contenidos en las categorías ya mencionadas.

En la escuela:

- $\quad$ Pláticas con el docente.

- $\quad$ Pláticas con director y otros administrativos.

- $\quad$ Asistencia a juntas de padres.

- $\quad$ Asistencia informal al aula de clase.

En el hogar:

- $\quad$ Restricción de televisión, videojuegos, tabletas, teléfonos y otros dispositivos electrónicos.

- Supervisión de tareas escolares a realizar en el hogar.

- $\quad$ Actividades de lectura durante el ocio.

- $\quad$ Asistencia a museos.

- Motivación y estímulo sobre logros académicos y aprendizaje.

- $\quad$ Pláticas sobre experiencia en la escuela. 


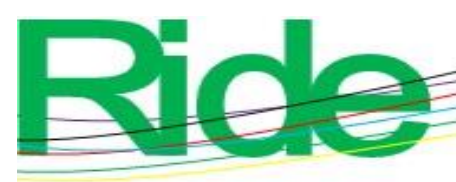

Revista Iberoamericana para la

Investigación y el Desarrollo Educativo

ISSN 2007 - 7467

\section{Metodología}

Se inició el proceso de revisión de la literatura reuniendo y analizando libros, capítulos, artículos de investigación, ponencias y tesis relacionados con la influencia de los padres en el rendimiento académico de alumnos de escuela primaria. Las siguientes bases de datos en línea se emplearon para buscar y recopilar estas fuentes: Scopus, base de datos bibliográfica de resúmenes y citas de artículos de revistas científicas de las áreas de tecnología, medicina y ciencias sociales, incluyendo artes y humanidades; Elsevier, la más grande editorial de acervo sobre medicina y literatura científica del mundo. ERIC es la mayor base de datos especializada en educación disponible en línea; PsycInfo, versión en línea de Psychological Abstracts que cubre artículos de revistas, capítulos de libros, libros, informes técnicos y disertaciones en psicología y aspectos psicológicos de disciplinas relacionadas; EBSCOhost, sistema en línea que proporciona acceso a índices periódicos o bases de datos de citas, resúmenes y artículos de texto completo de revistas. Además de estas bases de datos, el Sistema de Clasificación de Revistas Mexicanas de Ciencia y Tecnología del Consejo Nacional de Ciencia y Tecnología (Conacyt), Google Scholar y la Biblioteca Brigham en el Educational Testing Service se utilizaron para buscar y adquirir referencias específicas. Al respecto, el Sistema de Clasificación de Revistas Mexicanas de Ciencia y Tecnología del Conacyt agrupa las revistas reconocidas por su calidad evaluadas el año anterior en las diferentes áreas del conocimiento; Google Scholar es un sitio web que proporciona artículos revisados, libros, resúmenes y artículos de editoriales académicas, sociedades profesionales, universidades y otras organizaciones académicas; la Biblioteca Brigham en Educational Testing Service alberga colecciones completas de literatura educativa, psicológica, sociológica y de pruebas.

\section{Criterios de inclusión}

Se emplearon en la búsqueda de documentos completos las siguientes cadenas de texto: participación de los padres y rendimientos académico, la influencia de los padres y rendimientos académico, la influencia de la familia y rendimientos académico, el rendimiento académico en primaria, en español, inglés y francés. Para su inclusión en esta revisión, una publicación debía investigar la participación de los padres y su relación con el rendimiento académico de los alumnos de 6 a 12 años (nivel primaria); proporcionar descripciones claras de la estructura y las medidas de 


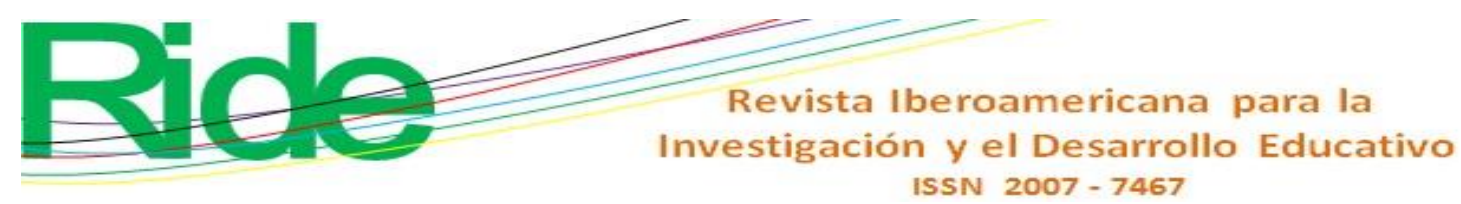

participación de los padres y el tipo de resultado académico; haberse publicado en el período 2011 y 2019. Se excluyeron estudios que investigaron, únicamente, los efectos de la participación de los padres en el comportamiento inadaptado de los estudiantes, como las publicaciones que incluyeron medidas de agresión, acoso escolar, delincuencia o depresión de los alumnos, debido a que esta revisión se centró en las expresiones de participación que los padres utilizan para fomentar el rendimiento académico de sus hijos.

La mayoría de los resultados arrojados por las bases de datos mencionadas fueron artículos de revistas, capítulos de libros e informes de investigación, seguidos por libros. Se excluyeron revisiones de literatura y estados del arte que no se combinaban con ningún análisis de los datos recabados para realizar nuevas conclusiones, además de estudios que no cumplieron con los criterios especificados. Estos criterios se utilizaron en una selección inicial de los resúmenes de las publicaciones. Si no había un resumen disponible, se examinó la publicación completa. Una primera selección resultó en la inclusión provisional de 184 publicaciones. Los 41 estudios que finalmente se obtuvieron después de una cuidadosa consideración y análisis reflejan todos los criterios de inclusión y exclusión.

\section{Resultados}

En este apartado se analizan y discuten las publicaciones que tratan sobre la influencia de los padres de familia en el aprovechamiento escolar de sus hijos en nivel primaria. Enseguida se muestra la clasificación de estos documentos. La tabla 1 clasifica las investigaciones de acuerdo con la fecha y lugar de publicación, autor y título del artículo, principal contribución al área de estudio, diseño utilizado en la investigación y tamaño de la muestra o cantidad de los sujetos de estudio $(N)$; aspectos listados de manera descendente por fecha de publicación. 


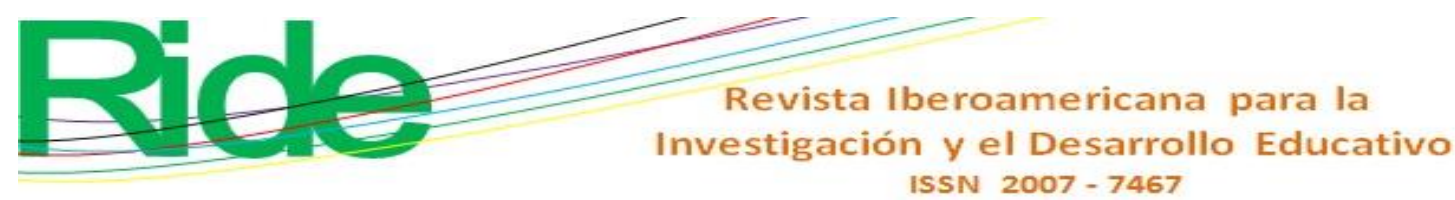

Tabla 1. Publicaciones sobre la participación de padres en el rendimiento académico de sus hijos en nivel primaria

\begin{tabular}{|c|c|c|c|c|}
\hline $\begin{array}{l}\text { Fecha y } \\
\text { lugar }\end{array}$ & Autor y título & Contribución & $\begin{array}{c}\text { Diseñode } \\
\text { estudio }\end{array}$ & $N$ \\
\hline 产 & $\begin{array}{l}\text { Reparaz y Sotés. } \\
\text { Parental involvement } \\
\text { in schools in Spain and } \\
\text { Germany: Evidence } \\
\text { from PISA } 2015\end{array}$ & $\begin{array}{l}\text { Analiza la participación de los } \\
\text { padres en las escuelas de Alemania } \\
\text { y España basándose en los } \\
\text { resultados del Programme for } \\
\text { International Student Assessment } \\
\text { (PISA) 2015. Los resultados } \\
\text { muestran los grandes esfuerzos } \\
\text { realizados en las escuelas para } \\
\text { involucrar a los padres, según los } \\
\text { directores entrevistados. Con } \\
\text { respecto al desempeño en ciencia, } \\
\text { se encuentra en España y en } \\
\text { Alemania que no todos los factores } \\
\text { analizados en relación con la } \\
\text { participación de los padres } \\
\text { favorecen un mayor logro. }\end{array}$ & $\begin{array}{c}\text { Descriptivo, ex } \\
\text { postfacto }\end{array}$ & $\begin{array}{l}540 \\
000\end{array}$ \\
\hline 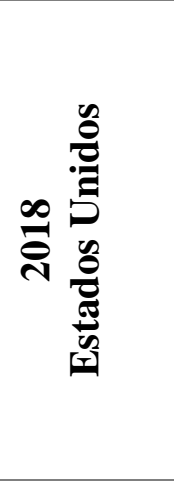 & $\begin{array}{c}\text { Dulay, Cheung, Reyes } \\
\text { y McBride. } \\
\text { Effects of parent } \\
\text { coaching on Filipino } \\
\text { children's numeracy, } \\
\text { language, and literacy } \\
\text { skills }\end{array}$ & $\begin{array}{l}\text { Se observaron evidencias de } \\
\text { transferencia entre dominios de la } \\
\text { capacitación en alfabetización } \\
\text { temprana a algunas habilidades de } \\
\text { cálculo numérico, así como } \\
\text { variaciones según el estado } \\
\text { socioeconómico, el nivel de } \\
\text { participación de los padres y la } \\
\text { asignación de maestros en el } \\
\text { programa. }\end{array}$ & Experimental & 578 \\
\hline 突 & $\begin{array}{l}\text { Guo, Lv, Zhou, Liu, } \\
\text { Liu, Jiang y Luo. } \\
\text { Gender differences in } \\
\text { how family income and } \\
\text { parental education } \\
\text { relate to reading } \\
\text { achievement in China: } \\
\text { The mediating role of } \\
\text { parental expectation } \\
\text { and parental } \\
\text { involvement }\end{array}$ & $\begin{array}{l}\text { Estos hallazgos sugieren un } \\
\text { proceso a través del cual los } \\
\text { factores de estatus socioeconómico } \\
\text { se relacionan con el desarrollo } \\
\text { académico de los niños e } \\
\text { identifican un contexto en el cual } \\
\text { estas asociaciones pueden diferir. } \\
\text { Se discuten las implicaciones } \\
\text { prácticas de estos hallazgos, junto } \\
\text { con posibles direcciones de } \\
\text { investigación futuras. }\end{array}$ & $\begin{array}{c}\text { Estudio } \\
\text { longitudinal }\end{array}$ & 598 \\
\hline 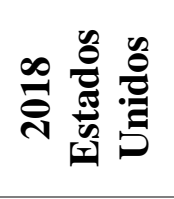 & $\begin{array}{c}\text { Schaeffer, Rozek, } \\
\text { Berkowitz, Levine, y } \\
\text { Beilock. }\end{array}$ & $\begin{array}{l}\text { Los niños de padres con mayores } \\
\text { niveles de matemáticas aprenden } \\
\text { menos matemáticas durante los } \\
\text { grados } 1 .^{\circ} \text { y } 3 .^{\circ} \text {, pero este no es el }\end{array}$ & Encuesta & 587 \\
\hline
\end{tabular}




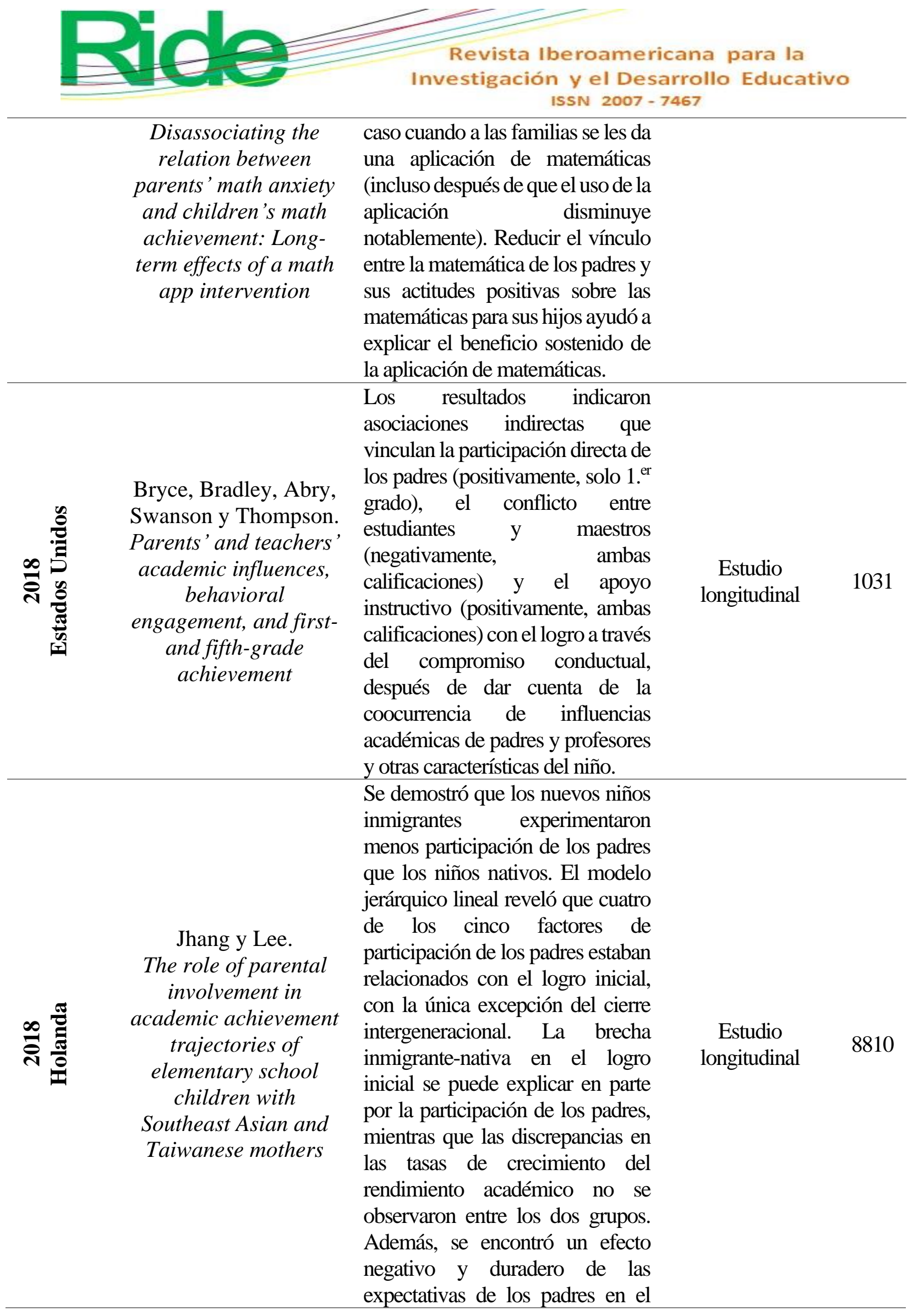




\begin{tabular}{|c|c|c|c|c|}
\hline & & $\begin{array}{l}\text { logro en la cultura confuciana, que } \\
\text { se caracteriza por un énfasis en la } \\
\text { importancia de la educación y los } \\
\text { estándares académicos más altos } \\
\text { establecidos por los padres chinos. }\end{array}$ & & \\
\hline 징 & $\begin{array}{l}\text { Criado y Gómez. } \\
\text { El mito de la dimisión } \\
\text { parental. Implicación } \\
\text { familiar, desigualdad } \\
\text { social y éxito escolar }\end{array}$ & $\begin{array}{l}\text { Numerosas políticas públicas, así } \\
\text { como docentes e investigadores, } \\
\text { atribuyen el menor rendimiento } \\
\text { escolar de las clases populares a la } \\
\text { falta de implicación parental y esta } \\
\text { a su menor valoración de la } \\
\text { escolaridad. Las clases populares } \\
\text { valoran y se implican en la } \\
\text { escolaridad filial, pero tienen } \\
\text { menos recursos para promoverla. }\end{array}$ & Encuesta & 2802 \\
\hline 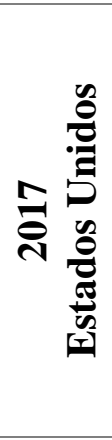 & $\begin{array}{l}\text { Borelli, Hong, } \\
\text { Rasmussen y Smiley. } \\
\text { Reflective functioning, } \\
\text { physiological } \\
\text { reactivity, and } \\
\text { overcontrol in } \\
\text { mothers: Links with } \\
\text { school-aged children's } \\
\text { reflective functioning }\end{array}$ & $\begin{array}{l}\text { Los hallazgos proporcionan } \\
\text { evidencia de una función } \\
\text { protectora de los padres y pueden } \\
\text { apuntar hacia la importancia de } \\
\text { promover el comportamiento } \\
\text { reflexivo en los programas de } \\
\text { intervención en nivel primaria para } \\
\text { reducir el control excesivo de los } \\
\text { padres hacia sus hijos. }\end{array}$ & Entrevista & 106 \\
\hline 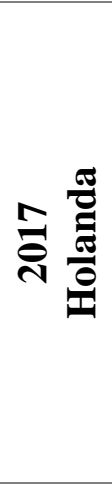 & $\begin{array}{c}\text { Park, Stone y } \\
\text { Holloway. } \\
\text { School-based parental } \\
\text { involvement as a } \\
\text { predictor of } \\
\text { achievement and } \\
\text { school learning } \\
\text { environment: An } \\
\text { elementary school- } \\
\text { level analysis }\end{array}$ & $\begin{array}{l}\text { Las escuelas con redes de } \\
\text { comunicación entre los padres eran } \\
\text { más propensas que otras escuelas a } \\
\text { tener porcentajes más altos de } \\
\text { estudiantes con logros altos en } \\
\text { matemáticas y lectura y es más } \\
\text { probable que muestren entornos de } \\
\text { aprendizaje más positivos. }\end{array}$ & $\begin{array}{c}\text { Estudio } \\
\text { longitudinal }\end{array}$ & 914 \\
\hline N兽 & $\begin{array}{l}\text { Loughlin-Presnal y } \\
\text { Bierman. } \\
\text { How do parent } \\
\text { expectations promote } \\
\text { child academic } \\
\text { achievement in early } \\
\text { elementary school? A } \\
\text { test of three mediators }\end{array}$ & $\begin{array}{l}\text { Existen asociaciones } \\
\text { longitudinales bidireccionales } \\
\text { entre las expectativas académicas } \\
\text { de los padres y los resultados } \\
\text { académicos de los niños. Los } \\
\text { comportamientos de aprendizaje } \\
\text { del niño mediaron en esta } \\
\text { asociación de primer a tercer } \\
\text { grado, mientras que la } \\
\text { competencia académica percibida }\end{array}$ & $\begin{array}{c}\text { Estudio } \\
\text { longitudinal }\end{array}$ & 356 \\
\hline
\end{tabular}




\begin{tabular}{|c|c|c|c|c|}
\hline & & $\begin{array}{l}\text { por el niño fue mediada de } \\
\text { segundo a quinto grado. }\end{array}$ & & \\
\hline 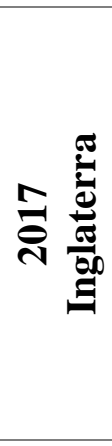 & $\begin{array}{l}\text { Park y Holloway. } \\
\text { The effects of school- } \\
\text { based parental } \\
\text { involvement on } \\
\text { academic achievement } \\
\text { at the child and } \\
\text { elementary school } \\
\text { level: A longitudinal } \\
\text { study }\end{array}$ & $\begin{array}{l}\text { Examina la influencia a largo plazo } \\
\text { de los padres sobre el rendimiento } \\
\text { de los estudiantes de primaria. Y } \\
\text { encuentra que su involucramiento } \\
\text { eleva el rendimiento en } \\
\text { matemáticas de los estudiantes, } \\
\text { pero solo el involucramiento muy } \\
\text { elevado se asoció con mejora en el } \\
\text { rendimiento en lectura. }\end{array}$ & $\begin{array}{c}\text { Estudio } \\
\text { longitudinal }\end{array}$ & $\begin{array}{c}21 \\
409\end{array}$ \\
\hline 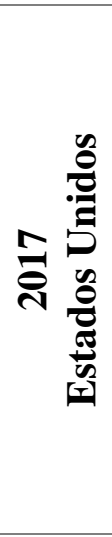 & $\begin{array}{l}\text { Tan, Kim, Baggerly, } \\
\text { Mahoney y Rice. } \\
\text { Beyond adoption } \\
\text { status: Post-adoptive } \\
\text { parental involvement } \\
\text { and children's reading } \\
\text { and math performance } \\
\text { from kindergarten to } \\
\text { first grade }\end{array}$ & $\begin{array}{l}\text { Los datos descriptivos no } \\
\text { mostraron diferencias grupales en } \\
\text { los puntajes de lectura, pero los } \\
\text { niños adoptados obtuvieron } \\
\text { calificaciones más bajas que los } \\
\text { niños no adoptados en } \\
\text { matemáticas. Sin embargo, se } \\
\text { demostró que el estado de } \\
\text { adopción no estaba relacionado } \\
\text { con los puntajes de lectura y } \\
\text { matemáticas. }\end{array}$ & $\begin{array}{c}\text { Estudio } \\
\text { longitudinal }\end{array}$ & $\begin{array}{c}14 \\
128\end{array}$ \\
\hline 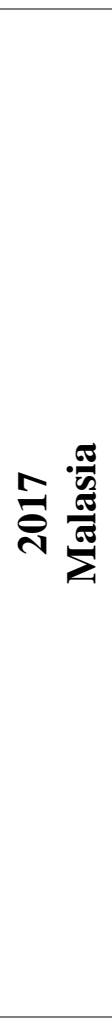 & $\begin{array}{c}\text { Kuan y Chuen. } \\
\text { A Study on Parental } \\
\text { Involvement and } \\
\text { Academic Achievement } \\
\text { in Elementary School } \\
\text { Students }\end{array}$ & $\begin{array}{l}\text { A través de un diseño de encuesta } \\
\text { cuantitativa se analizaron } 150 \\
\text { padres de estudiantes de primaria } \\
\text { en Johor Bahru. La correlación } r \text { de } \\
\text { Pearson se utilizó para medir la } \\
\text { relación entre la participación de } \\
\text { los padres y el rendimiento } \\
\text { académico del alumno. Sin } \\
\text { embargo, contrariamente a los } \\
\text { hallazgos previos, los resultados } \\
\text { mostraron que no hubo una } \\
\text { relación significativa entre la } \\
\text { participación de los padres y el } \\
\text { rendimiento académico del } \\
\text { estudiante ( } n=110, r=0.175, p> \\
0.05 \text { ). A pesar de la inconsistencia, } \\
\text { se encontró que existe una relación } \\
\text { significativa entre el constructo de } \\
\text { la autoeficacia de los padres y el } \\
\text { rendimiento académico del } \\
\text { alumno. }\end{array}$ & Encuesta & 150 \\
\hline
\end{tabular}




\section{Revista Iberoamericana para la Investigación y el Desarrollo Educativo ISSN 2007 - 7467}

\begin{tabular}{|c|c|c|c|c|}
\hline No & $\begin{array}{c}\text { Ucus, Garcia, } \\
\text { Esteraich y Raikes. } \\
\text { Predictors and } \\
\text { behavioural outcomes } \\
\text { of parental } \\
\text { involvement among } \\
\text { low-income families in } \\
\text { elementary schools, } \\
\text { United States }\end{array}$ & $\begin{array}{l}\text { El estudio destaca las } \\
\text { características del hogar y el } \\
\text { involucramiento de los padres de } \\
\text { bajos ingresos con sus hijos } \\
\text { estudiantes de quinto grado en su } \\
\text { educación y demuestra el papel } \\
\text { mediador de la de los progenitores } \\
\text { en la reducción de los problemas } \\
\text { de conducta de los niños. }\end{array}$ & Encuesta & 1354 \\
\hline 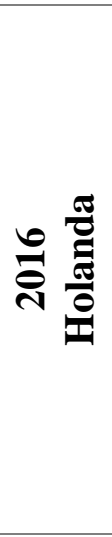 & $\begin{array}{c}\text { Tardif-Grenier y } \\
\text { Archambault. } \\
\text { Validation du } \\
\text { Questionnaire sur } \\
\text { l'implication parentale } \\
\text { dans le suivi scolaire } \\
\text { (QIPSS) chez des } \\
\text { parents d'élèves du } \\
\text { primaire en contexte } \\
\text { défavorisé et } \\
\text { pluriethnique }\end{array}$ & $\begin{array}{l}\text { Se generó un modelo de } \\
\text { participación de los padres que } \\
\text { comprende cinco dimensiones } \\
\text { para predecir el rendimiento } \\
\text { académico de los estudiantes. Los } \\
\text { hallazgos se discuten en } \\
\text { comparación de estudios previos } \\
\text { de participación de los padres. }\end{array}$ & Análisis factorial & 711 \\
\hline 串 & $\begin{array}{l}\text { Moon y Hofferth. } \\
\text { Parental involvement, } \\
\text { child effort, and the } \\
\text { development of } \\
\text { immigrant boys' and } \\
\text { girls' reading and } \\
\text { mathematics skills: A } \\
\text { latent difference score } \\
\text { growth model }\end{array}$ & $\begin{array}{l}\text { Se analizaron cómo las diferencias } \\
\text { en la participación de los padres, el } \\
\text { esfuerzo de niñas y niños y las } \\
\text { características y recursos } \\
\text { familiares contribuyen al } \\
\text { rendimiento de niños inmigrantes } \\
\text { desde el jardín de infantes hasta el } \\
\text { quinto grado. Se encontró que la } \\
\text { participación de los padres en el } \\
\text { hogar benefició las habilidades de } \\
\text { lectura y matemáticas de los niños } \\
\text { durante todos los primeros años de } \\
\text { escuela primaria, pero no tuvo el } \\
\text { mismo beneficio para las niñas. } \\
\text { Las asociaciones positivas de } \\
\text { participación de los padres y el } \\
\text { esfuerzo del niño con los puntajes } \\
\text { de las pruebas fueron mayores } \\
\text { durante los primeros años que en } \\
\text { los últimos años para los niños, } \\
\text { mientras que no hubo diferencias } \\
\text { en la asociación a lo largo del } \\
\text { tiempo para las niñas. }\end{array}$ & $\begin{array}{c}\text { Estudio } \\
\text { longitudinal }\end{array}$ & 2613 \\
\hline
\end{tabular}




\begin{tabular}{|c|c|c|c|c|}
\hline & & \multicolumn{3}{|c|}{$\begin{array}{l}\text { Revista Iberoamericana para la } \\
\text { Investigación y el Desarrollo Educativo } \\
\text { ISSN } 2007-7467\end{array}$} \\
\hline ชำ & $\begin{array}{l}\text { Lv, Zhou, Guo, Liu, } \\
\text { Liu y Luo. } \\
\text { The relationship } \\
\text { between academic } \\
\text { achievement and the } \\
\text { emotional well-being } \\
\text { of elementary school } \\
\text { children in China: The } \\
\text { moderating role of } \\
\text { parent-school } \\
\text { communication }\end{array}$ & $\begin{array}{l}\text { Este estudio investigó la relación } \\
\text { entre el logro académico y el } \\
\text { bienestar emocional (afecto } \\
\text { positivo y negativo) de los niños de } \\
\text { la escuela primaria en China y el } \\
\text { efecto moderador de la } \\
\text { comunicación entre padres y la } \\
\text { escuela sobre esta relación. El } \\
\text { compromiso de los padres con la } \\
\text { escuela afecta tanto los logros } \\
\text { académicos como el bienestar } \\
\text { subjetivo de los niños en China. }\end{array}$ & $\begin{array}{l}\text { Estudio } \\
\text { longitudinal }\end{array}$ & 419 \\
\hline 电 & $\begin{array}{l}\text { Ma, Shen, Krenn, Hu y } \\
\text { Yuan. } \\
\text { A Meta-Analysis of the } \\
\text { Relationship Between } \\
\text { Learning Outcomes } \\
\text { and Parental } \\
\text { Involvement During } \\
\text { Early Childhood } \\
\text { Education and Early } \\
\text { Elementary Education }\end{array}$ & $\begin{array}{l}\text { Los resultados del aprendizaje son } \\
\text { logros académicos, y los marcos de } \\
\text { participación de los padres miden } \\
\text { la participación familiar y el } \\
\text { desarrollo de asociaciones. La } \\
\text { relación (con el ajuste sobre los } \\
\text { marcos y las características del } \\
\text { estudio) indicó una fuerte y } \\
\text { positiva correlación (.509) entre los } \\
\text { resultados de aprendizaje y la } \\
\text { participación de los padres. }\end{array}$ & Metaanálisis & 46 \\
\hline 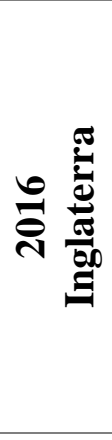 & $\begin{array}{l}\text { Tazouti y Jarlégan. } \\
\text { The mediating effects } \\
\text { of parental self- } \\
\text { efficacy and parental } \\
\text { involvement on the link } \\
\text { between family } \\
\text { socioeconomic status } \\
\text { and children's } \\
\text { academic achievement }\end{array}$ & $\begin{array}{l}\text { El presente estudio examinó la } \\
\text { hipótesis de que la autoeficacia de } \\
\text { los padres y su participación en la } \\
\text { educación de los niños median el } \\
\text { vínculo entre el estado } \\
\text { socioeconómico de la familia y el } \\
\text { rendimiento académico de los } \\
\text { niños. }\end{array}$ & $\begin{array}{l}\text { Ecuación } \\
\text { estructural }\end{array}$ & 203 \\
\hline 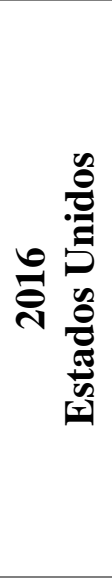 & $\begin{array}{c}\text { Jeynes. } \\
\text { A Meta-Analysis: The } \\
\text { Relationship Between } \\
\text { Parental Involvement } \\
\text { and African American } \\
\text { School Outcomes }\end{array}$ & $\begin{array}{l}\text { Se analiza la relación entre la } \\
\text { participación de los padres y el } \\
\text { rendimiento académico y el } \\
\text { comportamiento escolar de los } \\
\text { preescolares afroamericanos hasta } \\
\text { los estudiantes universitarios de } \\
\text { primer año de universidad. Existe } \\
\text { relación tanto para los estudiantes } \\
\text { más jóvenes (primaria) como para } \\
\text { los de mayor edad (estudiantes de } \\
\text { primer año de secundaria y } \\
\text { universitarios), así como para }\end{array}$ & Metaanálisis & 42 \\
\hline
\end{tabular}


ciertos componentes específicos de

la participación de los padres.

\begin{tabular}{|c|c|c|c|c|}
\hline n & $\begin{array}{c}\text { Jeynes. } \\
\text { A Meta-Analysis: The } \\
\text { Relationship Between } \\
\text { Father Involvement } \\
\text { and Student Academic } \\
\text { Achievement }\end{array}$ & $\begin{array}{l}\text { Se realizaron análisis estadísticos } \\
\text { para determinar el impacto general } \\
\text { y los componentes específicos de } \\
\text { la participación del padre. } \\
\text { También se examinaron los } \\
\text { posibles efectos diferenciales de la } \\
\text { participación paterna por raza. Los } \\
\text { resultados indican que la } \\
\text { asociación entre la participación } \\
\text { del padre y los resultados } \\
\text { educativos de los jóvenes en } \\
\text { general es estadísticamente } \\
\text { significativa. }\end{array}$ & Metaanálisis & 66 \\
\hline 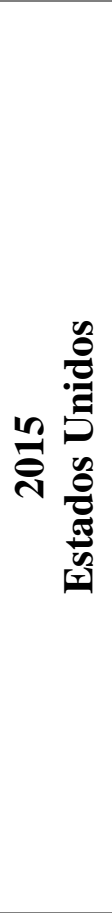 & $\begin{array}{c}\text { Silinskas, Kiuru, } \\
\text { Aunola, Lerkkanen y } \\
\text { Nurmi. } \\
\text { The developmental } \\
\text { dynamics of children's } \\
\text { academic performance } \\
\text { and mothers' } \\
\text { homework-related } \\
\text { affect and practices }\end{array}$ & $\begin{array}{l}\text { Cuanto mayor era la ayuda en las } \\
\text { tareas por las madres, más lento es } \\
\text { el desarrollo del rendimiento } \\
\text { académico de sus hijos desde el } \\
\text { grado } 1 \text { hasta el grado } 4 \text {. Esta } \\
\text { asociación negativa era cierta, } \\
\text { especialmente si las madres } \\
\text { percibían que sus hijos no eran } \\
\text { capaces de trabajar de forma } \\
\text { autónoma. Además, el buen } \\
\text { desempeño académico de los niños } \\
\text { en el grado } 1 \text { predijo la percepción } \\
\text { de las madres sobre la capacidad } \\
\text { del niño para ser autónomo y el } \\
\text { efecto positivo en situaciones de } \\
\text { tarea más adelante, mientras que el } \\
\text { bajo desempeño predijo el efecto } \\
\text { negativo, la ayuda y el monitoreo } \\
\text { de las madres. }\end{array}$ & $\begin{array}{c}\text { Estudio } \\
\text { longitudinal }\end{array}$ & 2261 \\
\hline 疍 & $\begin{array}{l}\text { Núñez, Suárez, } \\
\text { Rosário, Vallejo, Valle } \\
\text { y Epstein. } \\
\text { Relationships between } \\
\text { perceived parental } \\
\text { involvement in } \\
\text { homework, student } \\
\text { homework behaviors, } \\
\text { and academic }\end{array}$ & $\begin{array}{l}\text { El comportamiento en las tareas de } \\
\text { los estudiantes, la participación } \\
\text { percibida de los padres y el } \\
\text { rendimiento académico están } \\
\text { significativamente relacionados. } \\
\text { Sin embargo, los resultados varían } \\
\text { según el nivel de grado de los } \\
\text { estudiantes. La participación de los } \\
\text { padres en las tareas se relaciona es }\end{array}$ & $\begin{array}{c}\text { Ecuaciones } \\
\text { estructurales }\end{array}$ & 1683 \\
\hline
\end{tabular}




\begin{tabular}{|c|c|c|c|c|}
\hline & $\begin{array}{c}\text { achievement: } \\
\text { differences among } \\
\text { elementary, junior } \\
\text { high, and high school } \\
\text { students }\end{array}$ & $\begin{array}{l}\text { más fuerte en secundaria que en } \\
\text { primaria; y a pesar de que los } \\
\text { comportamientos en las tareas de } \\
\text { los estudiantes están relacionados } \\
\text { con el rendimiento académico en } \\
\text { cada nivel escolar, la dirección y la } \\
\text { magnitud de las relaciones varían. } \\
\text { Específicamente, la relación entre } \\
\text { la participación percibida de los } \\
\text { padres y el rendimiento académico } \\
\text { es más fuerte en la escuela } \\
\text { secundaria y preparatoria que en la } \\
\text { escuela primaria. }\end{array}$ & & \\
\hline$\frac{n}{8}$ & $\begin{array}{l}\text { Mante, Awereh y } \\
\text { Kumea. } \\
\text { Effects of parental } \\
\text { involvement on } \\
\text { academic performance } \\
\text { of pupils: A Case Study } \\
\text { at Adukrom Methodist } \\
\text { Primary School }\end{array}$ & $\begin{array}{l}\text { La investigación se centró en } 26 \\
\text { estudiantes, } 11 \text { niños y } 15 \text { niñas, de } \\
\text { los } 50 \text { estudiantes de la clase. } 69 \% \\
\text { de los alumnos afirmaron que con } \\
\text { la participación de sus padres en } \\
\text { sus actividades académicas se } \\
\text { esfuerzan más por mejorar el } \\
\text { rendimiento, mientras que } 19 \% \\
\text { afirma que la participación de sus } \\
\text { padres no afecta su rendimiento. } 11 \\
\text { padres (79 \%) indicaron que } \\
\text { sentían que estaban involucrados } \\
\text { en la educación de sus hijos y } 5 \text { de } \\
\text { estos } 11 \text { padres (43 \%) afirmaron } \\
\text { que se sentían muy involucrados. } \\
\text { Solo un padre (7\%) indicó que no } \\
\text { estaba muy involucrado en la } \\
\text { educación de su hijo. En } \\
\text { conclusión, la participación de los } \\
\text { padres en las actividades } \\
\text { académicas de los alumnos afecta } \\
\text { su rendimiento académico. }\end{array}$ & $\begin{array}{c}\text { Cuestionario, } \\
\text { entrevista, } \\
\text { observación, } \\
\text { encuesta }\end{array}$ & 26 \\
\hline n & $\begin{array}{c}\text { Fajoju, Aluede y } \\
\text { Ojugo. } \\
\text { Parental involvement } \\
\text { as a correlate of } \\
\text { academic achievement } \\
\text { of primary school } \\
\text { pupils in Edo State, } \\
\text { Nigeria }\end{array}$ & $\begin{array}{l}\text { La participación de los padres } \\
\text { influye en logros académicos en } \\
\text { tres asignaturas principales, } \\
\text { Lengua Inglesa, Matemáticas y } \\
\text { Ciencias Integradas, en la escuela } \\
\text { primaria y que cuanto mayor sea la } \\
\text { participación de los padres, mayor } \\
\text { será el rendimiento de los alumnos } \\
\text { en las tres materias básicas. Con } \\
\text { base en estos hallazgos, se }\end{array}$ & Ex post facto & 1895 \\
\hline
\end{tabular}




\begin{tabular}{|c|c|c|c|c|}
\hline & & $\begin{array}{l}\text { recomendó que los padres estén } \\
\text { más involucrados en la educación } \\
\text { de sus hijos y que controlen la } \\
\text { asistencia de sus hijos a la escuela. } \\
\text { Además, los consejeros escolares y } \\
\text { psicólogos educativos deben } \\
\text { emplear diversos enfoques para } \\
\text { lograr una mayor participación de } \\
\text { los padres en sus escuelas. }\end{array}$ & & \\
\hline 经 & $\begin{array}{c}\text { Ramírez, Quintero y } \\
\text { Jaramillo. Formación } \\
\text { en el trabajo con } \\
\text { familias para la } \\
\text { educación de la } \\
\text { primera infancia }\end{array}$ & $\begin{array}{l}\text { Se aborda la categoría Entorno } \\
\text { familiar y su articulación con la } \\
\text { institución educativa. El hallazgo } \\
\text { más importante es la } \\
\text { desarticulación entre la formación } \\
\text { profesional y las demandas del } \\
\text { contexto sociocultural en relación } \\
\text { con el apoyo familiar. }\end{array}$ & Estudio de caso & 47 \\
\hline n & $\begin{array}{l}\text { Maloney, Ramirez, } \\
\text { Gunderson, Levine y } \\
\text { Beilock. } \\
\text { Intergenerational } \\
\text { Effects of Parents' } \\
\text { Math Anxiety on } \\
\text { Children's Math } \\
\text { Achievement and } \\
\text { Anxiety }\end{array}$ & $\begin{array}{l}\text { En este estudio de campo de niños } \\
\text { en primer y segundo grado se } \\
\text { exploró cómo la ansiedad de los } \\
\text { padres sobre las matemáticas se } \\
\text { relaciona con el rendimiento de sus } \\
\text { hijos en esta ciencia. Cuando los } \\
\text { padres están más ansiosos por las } \\
\text { matemáticas, sus hijos aprenden } \\
\text { significativamente menos en esta } \\
\text { rama del saber durante el año } \\
\text { escolar y tienen más ansiedad al } \\
\text { final del mismo, pero solo si los } \\
\text { padres ansiosos informan que } \\
\text { proporcionan ayuda frecuente con } \\
\text { las tareas de matemáticas. }\end{array}$ & $\begin{array}{l}\text { Investigación de } \\
\text { campo }\end{array}$ & 529 \\
\hline 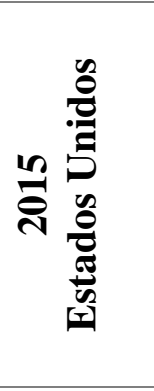 & $\begin{array}{c}\text { Benner y Yan. } \\
\text { Classroom } \\
\text { Race/Ethnic } \\
\text { Composition, Family- } \\
\text { School Connections, } \\
\text { and the Transition to } \\
\text { School }\end{array}$ & $\begin{array}{l}\text { Para los estudiantes con más } \\
\text { representación étnica/racial en sus } \\
\text { aulas, una mayor diversidad } \\
\text { étnica/racial promovió una mayor } \\
\text { participación de los padres, lo que } \\
\text { a su vez fomentó las habilidades } \\
\text { interpersonales de los niños y el } \\
\text { rendimiento en lectura. }\end{array}$ & $\begin{array}{c}\text { Estudio } \\
\text { longitudinal }\end{array}$ & $\begin{array}{c}13 \\
970\end{array}$ \\
\hline$\stackrel{n}{\frac{n}{8}}$ & $\begin{array}{l}\text { Cunha, Rosário, } \\
\text { Macedo, Nunes, } \\
\text { Fuentes y Pinto. } \\
\text { Parents' conceptions } \\
\text { of their homework }\end{array}$ & $\begin{array}{l}\text { Los padres presentan una } \\
\text { perspectiva positiva de su } \\
\text { implicación en los deberes } \\
\text { escolares y el aprendizaje } \\
\text { académico. Los datos obtenidos }\end{array}$ & Entrevista & 32 \\
\hline
\end{tabular}


involvement in indican que los padres pueden

elementary school implicarse para promocionar la autonomía de los niños; controlar el aprendizaje y sus resultados; y aportar apoyo motivacional $\mathrm{y}$ emocional (principalmente en presencia de dificultades).

La investigación estima una serie de modelos jerárquicos para evaluar los efectos directos e indirectos de la participación de los

McNeal.
Parent Involvement,
Academic Achievement
and the Role of Student
An
Attitudes and
Behaviors as
Mediators

Castro, Expósito, Lizasoain, López y Navarro.

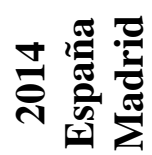
Participación familiar y rendimiento académico. Una sintesis meta-analítica padres en la actitud, el comportamiento y los resultados académicos de los hijos. Los hallazgos confirman que las prácticas de participación padrehijo y padres-escuela influyen de manera diferente en las actitudes y comportamientos de los estudiantes, lo que indirectamente afecta el rendimiento del alumno, en diversos grados.

No parece que estén vinculados al rendimiento conductas familiares como revisión de tareas o visitas a la escuela. La necesidad de apoyo para alumnos por parte de los papás se da cuando el discente presenta algún tipo de dificultad de aprendizaje, de comportamiento, de socialización, etc.

Se analizaron los efectos adversos de la crianza insensible temprana de las madres en el funcionamiento académico de los niños, lo cual puede ser compensado por la participación posterior de los padres en los niños. Aunque la insensibilidad de las madres prefirió el funcionamiento académico disminuido de los niños cuando la participación de los padres fue relativamente baja, no lo hizo cuando la participación de los padres fue promedio o superior 


\section{Revista Iberoamericana para la Investigación y el Desarrollo Educativo ISSN 2007 - 7467}

\begin{tabular}{|c|c|c|c|c|}
\hline ๙ & $\begin{array}{l}\text { Rafiq, Fatima, Sohail, } \\
\text { Saleem y Khan. } \\
\text { Parental Involvement } \\
\text { and Academic } \\
\text { Achievement; A Study } \\
\text { on Secondary School } \\
\text { Students of Lahore, } \\
\text { Pakistan }\end{array}$ & $\begin{array}{l}\text { En un estudio cuantitativo con } 150 \\
\text { estudiantes de } 4 \text { secundarias } \\
\text { públicas y privadas de Pakistán, se } \\
\text { encontró que la participación de los } \\
\text { padres tiene un efecto significativo } \\
\text { en un mejor rendimiento } \\
\text { académico de sus hijos. }\end{array}$ & Encuesta & 150 \\
\hline 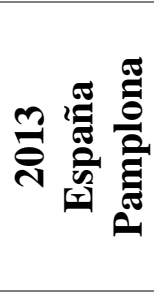 & $\begin{array}{l}\text { López y Ordóñez. } \\
\text { La vida en familia }\end{array}$ & $\begin{array}{l}\text { La educación es una labor básica } \\
\text { de los papás. } 90 \% \text { de los } \\
\text { encuestados en este estudio afirma } \\
\text { el protagonismo que deben tener } \\
\text { los progenitores en la educación de } \\
\text { sus hijos. }\end{array}$ & Encuesta & 1500 \\
\hline m. & $\begin{array}{c}\text { Ojeda, Lima y } \\
\text { González. } \\
\text { Papá y mamá: dos } \\
\text { maneras de ver la } \\
\text { vida ... y una misma } \\
\text { educación }\end{array}$ & $\begin{array}{l}\text { A una mayor intervención de la } \\
\text { familia, sin importar la escolaridad } \\
\text { del hijo, mayores son las } \\
\text { habilidades que adquiere, que } \\
\text { pueden ser indicadores apropiados } \\
\text { de logro escolar. Se encontraron } \\
\text { diferencias estadísticamente } \\
\text { significativas entre el padre y la } \\
\text { madre y la intervención en cuanto } \\
\text { a las fortalezas interpersonales de } \\
\text { los hijos. }\end{array}$ & Encuesta & 180 \\
\hline 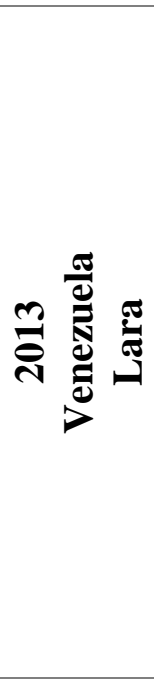 & $\begin{array}{l}\text { Silva y Tabernero. } \\
\text { Escuela y familia: } \\
\text { Alianza estratégica } \\
\text { para la prevención del } \\
\text { maltrato infantil }\end{array}$ & $\begin{array}{l}\text { Esta investigación tiene como } \\
\text { propósito diseñar acciones para } \\
\text { evitar el maltrato a menores } \\
\text { fortaleciendo el vínculo escuela- } \\
\text { familia, debido a que este } \\
\text { problema social socava el } \\
\text { rendimiento académico. Como } \\
\text { resultado del estudio se evidenció } \\
\text { la nula capacitación del docente } \\
\text { respecto al maltrato infantil, hecho } \\
\text { realmente alarmante, dado que si } \\
\text { no se posee conocimiento sobre } \\
\text { esta problemática social no se } \\
\text { puede prevenir. }\end{array}$ & Descriptivo & 88 \\
\hline 궁 & $\begin{array}{c}\text { Barajas. } \\
\text { La educación, la } \\
\text { familia y el derecho } \\
\text { familiar, como } \\
\text { solución al problema }\end{array}$ & $\begin{array}{l}\text { En esta tesis se concluye, entre } \\
\text { otras cosas, que en la familia } \\
\text { moderna ya no subsiste con el } \\
\text { mismo rigor el antiguo lazo de } \\
\text { unión más allá de padre-madre- } \\
\text { hijos-nietos. Tambiénque estas, las }\end{array}$ & Documental & N/A \\
\hline
\end{tabular}




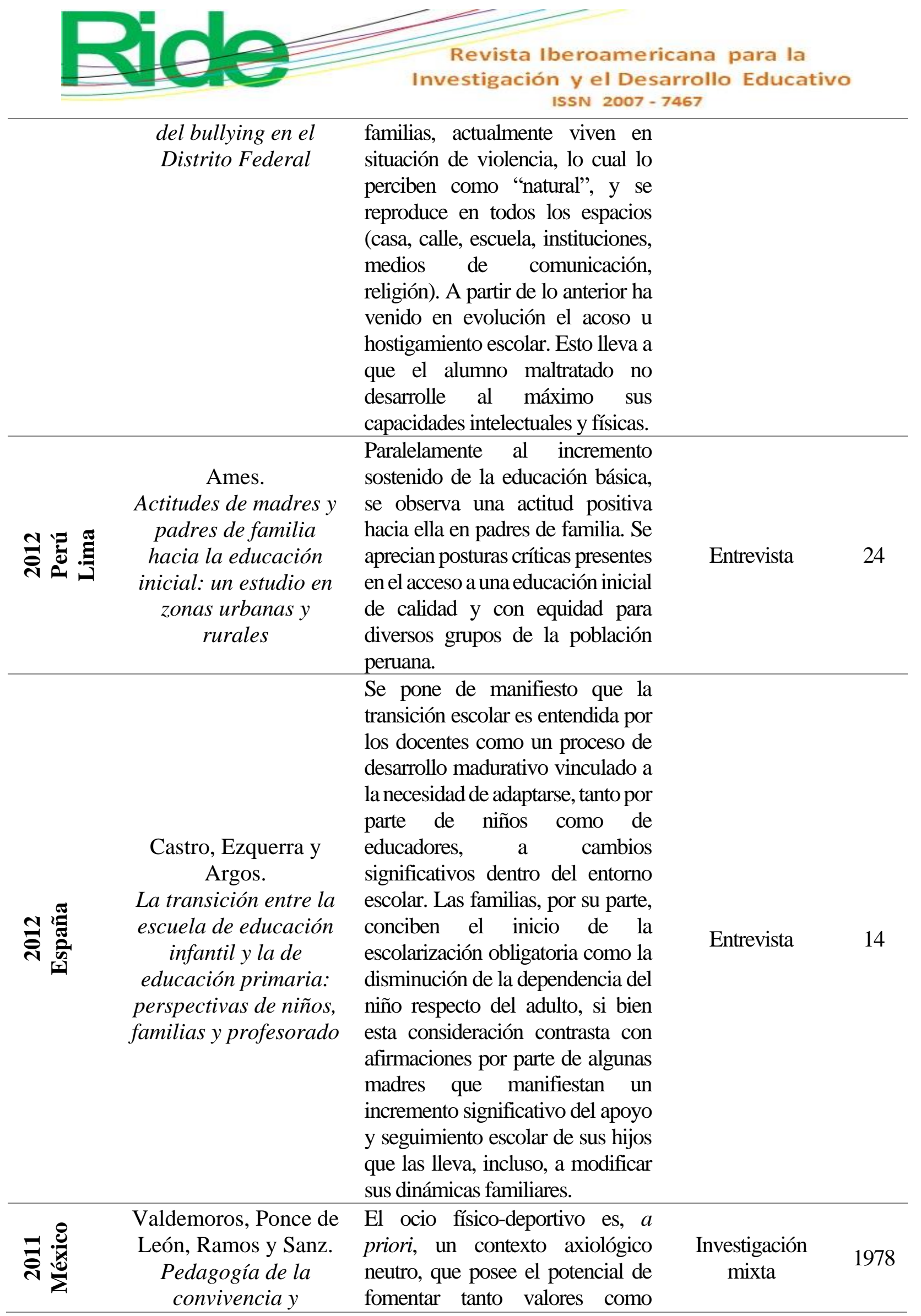




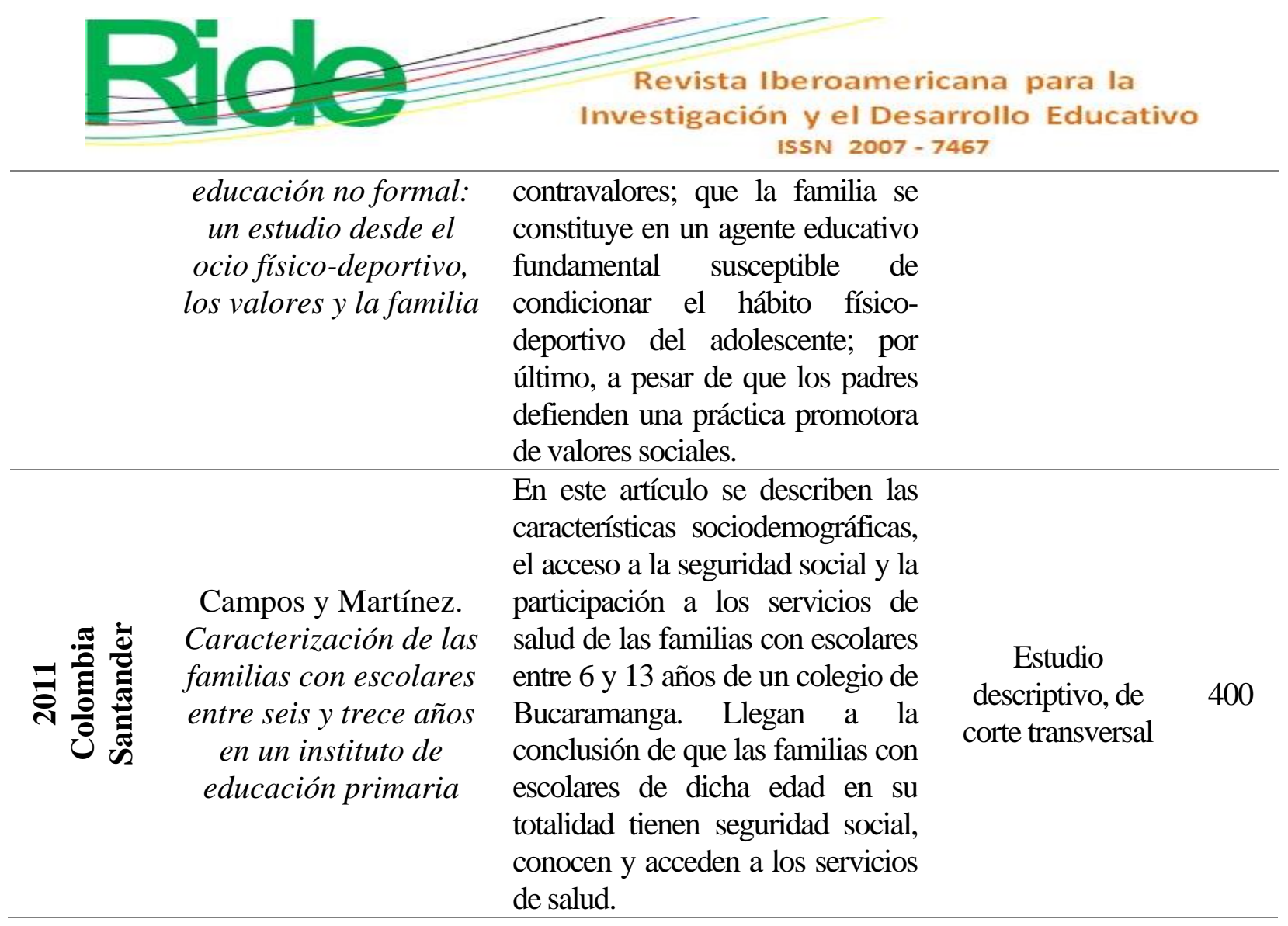

\section{Tendencias futuras}

Después del análisis realizado en la sección anterior, puede decirse que la tendencia marcada en las publicaciones va orientada a revalorizar el papel que desempeña la familia como soporte en la educación de los alumnos, vínculo que es considerado cada vez más en las investigaciones que comprenden el tema del aprendizaje y educación básica. Además, en términos generales, todo indica que los autores se enfocan en la evolución social a través de la academia y ponen como factor principal el papel de los padres y la familia. Las tendencias se inclinan a dejar atrás la idea de que únicamente la escuela y sus maestros son los responsables del quehacer educativo. 


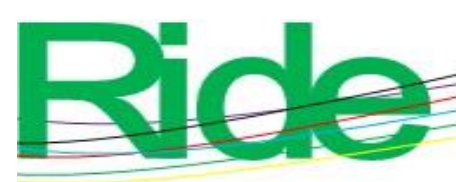

Revista Iberoamericana para la Investigación y el Desarrollo Educativo ISSN 2007 - 7467

\section{Conclusiones}

Al indagar sobre el tema y consultar los autores que se han interesado sobre la línea argumental que se maneja en esta investigación, se concluye que es amplio el interés que levanta la relación entre educación y padres de familia y su papel en el rendimiento académico de los niños, no solo en el país en que se desarrolla ni en un único contexto, sino en todas aquellas sociedades en que se interesen en los porqués de las tramas educativas. Resulta interesante saber que desde diferentes perspectivas, condiciones y circunstancias se resalta la importancia de la influencia que deriva la familia dentro de la educación formal e informal, considerándola punto central o definitivo para el rumbo que tomará el individuo en su formación durante edades tempranas y la adolescencia.

Hubo coincidencia entre las conclusiones a las que se llega en esta investigación con los diferentes investigadores analizados aquí: como el papel determinante de la familia en la medida en que apoye y estimule al alumno; el valor social que aporta la familia en un enfoque integral para la formación del individuo; el factor motivacional de vital importancia que representan padre y madre; la repercusión de un mal trato hacia el niño por sus padres; el niño representa un reflejo de las actitudes que hay en casa.

Para finalizar es digno de mencionar algo que no se consideró en este trabajo: la educación escolar desde casa y la educación o comunicación entre padres e hijos a través de medios digitales. La primera es poco común como enseñanza en México, pero los autores que abordan dicha temática, en su mayoría, hablan de buenos resultados obtenidos en estas modalidades. Respecto a la segunda, ninguna de las publicaciones analizadas aquí menciona estas herramientas, mucho menos las consideran como instrumentos de apoyo de los padres hacia el quehacer académico de sus hijos. 


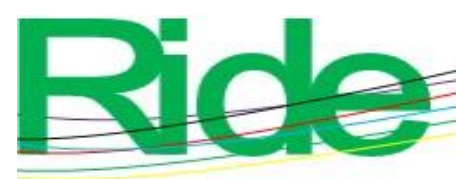

Revista Iberoamericana para la

Investigación y el Desarrollo Educativo

ISSN 2007 - 7467

\section{Referencias}

Ames, P. (2012). Actitudes de madres y padres de familia hacia la educación inicial: un estudio en zonas urbanas y rurales. Revista Educación, 21(40), 1-20. Recuperado de http://revistas.pucp.edu.pe/index.php/educacion/article/view/2500.

Backoff, E. (30 de marzo de 2016). Estudiantes de bajo rendimiento en México. El Universal. Recuperado de https://www.eluniversal.com.mx/entrada-de-opinion/articulo/eduardobackhoff-escudero/nacion/2016/03/30/estudiantes-de-bajo.

Baker, A. J. L. y Soden, L.M. (1998). The challenges of parent involvement research. ERIC/CUE Digest, (134), 1-6. Recuperado de https://eric.ed.gov/?id=ED419030.

Barajas, E. (2012). La educación, la familia y el derecho familiar, como solución al problema del bullying en el Distrito Federal. (tesis de licenciatura). Universidad Nacional Autónoma de México, Ciudad de México. Recuperado de http://132.248.9.195/ptd2015/anteriores/0685640/Index.html.

Benner, A. D. and Yan, N. (2015). Classroom Race/Ethnic Composition, Family-School Connections, and the Transition to School. Applied Developmental Science, 19(3), 127-138. Retrieved from https://doi.org/10.1080/10888691.2014.983028.

Borelli, J. L., Hong, K., Rasmussen, H. F. and Smiley, P. A. (2017). Reflective functioning, physiological reactivity, and overcontrol in mothers: Links with school-aged children's reflective functioning. Developmental Psychology, 53(9), 1680-1693. Retrieved from http://dx.doi.org/10.1037/dev0000371.

Bryce, C. I., Bradley, R. H., Abry, T., Swanson, J. and Thompson, M.S. (2018). Parents' and teachers' academic influences, behavioral engagement, and first- and fifth-grade achievement. School Psychology Quarterly. Advance online publication. Retrieved from http://dx.doi.org/10.1037/spq0000297.

Campos, M. S. y Martínez, A. (2011). Caracterización de las familias con escolares entre seis y trece años en un instituto de educación primaria. Revista Cuidarte, 2(1), 105-113. Recuperado de https://revistacuidarte.udes.edu.co/index.php/cuidarte/article/view/45/49.

Castro, A., Ezquerra, P. y Argos, J. (2012). La transición entre la Escuela de Educación Infantil y la de Educación Primaria: perspectivas de niños, familias y profesorado. Revista Española 


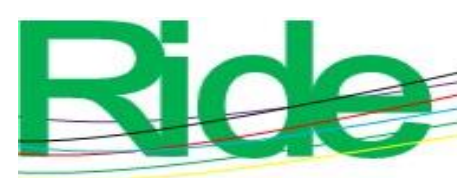

Revista Iberoamericana para la

Investigación y el Desarrollo Educativo

ISSN 2007 - 7467

de Pedagogía, 70(253), 537-552. Recuperado de https://revistadepedagogia.org/wpcontent/uploads/2012/09/253-08.pdf.

Castro, M., Expósito, E., Lizasoain, L., López, E. y Navarro, E. (2014). Participación familiar y rendimiento académico. Una síntesis meta-analítica. En Ministerio de Educación, Cultura y Deporte (ed.), La participación de las familias en la educación escolar. Mirando al futuro (pp. 83-102). España: Secretaría General Técnica.

Criado, E. y Gómez, B. (2017). El mito de la dimisión parental. Implicación familiar, desigualdad social y éxito escolar. Cuadernos de Relaciones Laborales, 35(2), 305-325. Recuperado de https://doi.org/10.5209/CRLA.56777.

Cunha, J., Rosário, P., Macedo, L., Nunes, A. R., Fuentes, S. and Pinto, R. (2015). Parents' conceptions of their homework involvement in elementary school. Psicothema, 27(2), 159165. Retrieved from https://doi.org/10.7334/psicothema2014.210.

Dulay, K. M., Cheung, S. K., Reyes, P. and McBride, C. (2018). Effects of parent coaching on Filipino children's numeracy, language, and literacy skills. Journal of Educational Psychology. Advance online publication. Retrieved from http://dx.doi.org/10.1037/edu0000315.

Epstein, J. L. (1991). Effects on student achievement of teachers' practices of parent involvement. En Silvern, S.B. (ed.), Advances in reading/language research: A research annual, Vol. 5. Literacy through family, community, and school interaction (pp. 261-276). Estados Unidos: Elsevier Science/JAI Press. Retrieved from https://psycnet.apa.org/record/1998-07316011.

Epstein, J. L. y Dauber, S. L. (1991). School Programs and Teacher Practices of Parent Involvement in Inner-City Elementary and Middle Schools. The Elementary School Journal, 91(3), 289305. Retrieved from https://doi.org/10.1086/461656.

Fajoju, S. A., Aluede, O. and Ojugo, A. I. (2015). Parental involvement as a correlate of academic achievement of primary school pupils in Edo State, Nigeria. Research in Education, 95(1), 33-43. Retrieved from https://doi.org/10.7227/RIE.0023.

Gómez, M. E. (2017). Panorama del sistema educativo mexicano desde la perspectiva de las políticas públicas. Innovación educativa, 17(74), 143-163. Recuperado de 


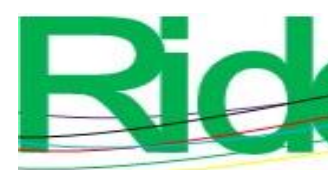

Revista Iberoamericana para la

Investigación y el Desarrollo Educativo

ISSN $2007-7467$

http://www.scielo.org.mx/scielo.php?script=sci_arttext\&pid=S1665-

$26732017000200143 \& \operatorname{lng}=$ es\&tlng=es.

Guo, X., Lv, B., Zhou, H., Liu, C., Liu, J., Jiang, K. and Luo, L. (2018). Gender differences in how family income and parental education relate to reading achievement in China: The mediating role of parental expectation and parental involvement. Frontiers in Psychology, 9(783). Retrieved from https://doi.org/10.3389/fpsyg.2018.00783.

Halle, T. G., Kurtz-Costes, B. and Mahoney, J. L. (1997). Family influences on school achievement in low-income, African American children. Journal of Educational Psychology, 89(3), 527 537. Retrieved from http://dx.doi.org/10.1037/0022-0663.89.3.527.

Instituto Nacional para la Evaluación de la Educación [INEE]. (2018). La educación obligatoria en México. México: Instituto Nacional para la Evaluación de la Educación. Recuperado de https://www.inee.edu.mx/portalweb/informe2018/04_informe/capitulo_070305.html.

Jeynes, W. H. (2015). A Meta-Analysis: The Relationship Between Father Involvement and Student Academic Achievement. Urban Education 50(4), 387-423. Retrieved from https://doi.org/10.1177/0042085914525789.

Jeynes, W. H. (2016). A Meta-Analysis: The Relationship Between Parental Involvement and African American School Outcomes. Journal of Black Studies, 47(3), 195-216. Retrieved from https://doi.org/10.1177/0021934715623522.

Jhang, F. and Lee, Y. (2018). The role of parental involvement in academic achievement trajectories of elementary school children with Southeast Asian and Taiwanese mothers. International Journal of Educational Research 89, 68-79. Retrieved from http://dx.doi.org/10.1016/j.ijer.2017.09.003.

Keith, T. Z., Reimers, T. M., Fehrmann, P. G., Pottebaum, S. M. and Aubey, L. W. (1986). Parental involvement, homework, and TV time: Direct and indirect effects on high school achievement. Journal of Educational Psychology, 78(5), 373-380. Retrieved from http://dx.doi.org/10.1037/0022-0663.78.5.373.

Kuan, N. W. and Chuen, T. W. (2017). A Study on Parental Involvement and Academic Achievement in Elementary School Students. Sains Humanika. 9(3-2), 45-50. Retrieved from https://sainshumanika.utm.my/index.php/sainshumanika/article/view/1272. 


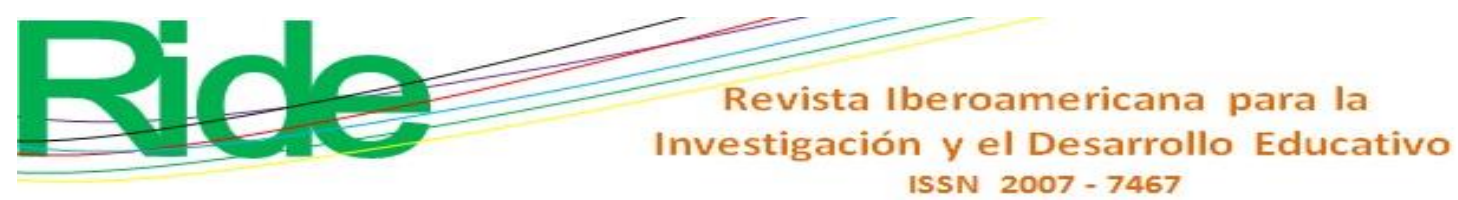

López, D. y Ordóñez, M. E. (2013). La vida en familia. En Montoro, C. (ed.), La familia, recurso de la sociedad (pp. 31-63). Pamplona, España: Universidad de Navarra.

Loughlin-Presnal, J. and Bierman, K. L. (2017). How do parent expectations promote child academic achievement in early elementary school? A test of three mediators. Developmental Psychology, 53(9), 1694-1708. Retrieved from http://dx.doi.org/10.1037/dev0000369.

Lv, B., Zhou, H., Guo, X., Liu, C., Liu, Z. and Luo, L. (2016). The relationship between academic achievement and the emotional well-being of elementary school children in China: The moderating role of parent-school communication. Frontiers in Psychology, 7(948). Retrieved from https://doi.org/10.3389/fpsyg.2016.00948.

Ma, X., Shen, J., Krenn, H.Y., Hu, S. and Yuan, J. (2016). A Meta-Analysis of the Relationship Between Learning Outcomes and Parental Involvement During Early Childhood Education and Early Elementary Education. Educational Psychology Review, 28(4), 771-801. Retrieved from https://doi.org/10.1007/s10648-015-9351-1.

Maloney, E. A., Ramirez, G., Gunderson, E. A., Levine, S. C. and Beilock, S. L. (2015). Intergenerational Effects of Parents' Math Anxiety on Children's Math Achievement and Anxiety. Psychological Science, 26(9), 1480-1488. Retrieved from https://doi.org/10.1177/0956797615592630.

Mante, F. A., Awereh, E. O. and Kumea, A. O. (2015). Effects of parental involvement on academic performance of pupils: A Case Study at Adukrom Methodist Primary School. Basic Research Journal of Education Research Review, 4(1), 1-7. Retrieved from http://basicresearchjournals.org/education/abstract/Mante\%20et\%20al.html.

McNeal, R. B. (2014). Parent Involvement, Academic Achievement and the Role of Student Attitudes and Behaviors as Mediators. Universal Journal of Educational Research 2(8), 564-576. Retrieved from https://doi.org/10.13189/ujer.2014.020805.

Monti, J. D., Pomerantz, E. M. and Roisman, G. I. (2014). Can parents' involvement in children's education offset the effects of early insensitivity on academic functioning? Journal of Educational Psychology, 106(3), 859-869. Retrieved from http://dx.doi.org/10.1037/a0035906. 
Moon, U. J. and Hofferth, S. L. (2016). Parental involvement, child effort, and the development of immigrant boys' and girls' reading and mathematics skills: A latent difference score growth model. Learning and Individual Differences, 47, 136-144. Retrieved from https://doi.org/10.1016/j.lindif.2016.01.001.

Núñez, J. C., Suárez, N., Rosário, P., Vallejo, G., Valle, A. and Epstein, J. L. (2015). Relationships between perceived parental involvement in homework, student homework behaviors, and academic achievement: differences among elementary, junior high, and high school students. Metacognition and Learning, 10(3), 375-406. Retrieved from https://doi.org/10.1007/s11409-015-9135-5.

Ojeda, A., Lima, L. y González, R. (2013). Papá y mamá: dos maneras de ver la vida... y una misma educación. Enseñanza e investigación en psicología, 18(1). Recuperado de https://www.redalyc.org/pdf/292/29228948004.pdf.

Park, S. and Holloway, S. D. (2017). The effects of school-based parental involvement on academic achievement at the child and elementary school level: A longitudinal study. The Journal of Educational Research, 110(1), 1-16. Retrieved from https://doi.org/10.1080/00220671.2015.1016600.

Park, S., Stone, S. and Holloway, S. D. (2017). School-based parental involvement as a predictor of achievement and school learning environment: An elementary school-level analysis. Children and Youth Services Review, 82(2017), 195-206. Retrieved from http://dx.doi.org/10.1016/j.childyouth.2017.09.012.

Rafiq, H. M. W., Fatima, T., Sohail, M. M., Saleem, M. and Khan, M.A. (2013). Parental Involvement and Academic Achievement; A Study on Secondary School Students of Lahore, Pakistan. International Journal of Humanities and Social Science, 3(8), 209-223. Retrieved from https://www.ijhssnet.com/journals/Vol_3_No_8_Special_Issue_April_2013/22.pdf.

Ramírez, L., Quintero, S. y Jaramillo, B. (2015). Formación en el trabajo con familias para la educación de la primera infancia. Zona Próxima, (22). Recuperado de http://rcientificas.uninorte.edu.co/index.php/zona/article/view/5832/6879. 
Reparaz, C. and Sotés, M. (2019). Parental involvement in schools in Spain and Germany: Evidence from PISA 2015. International Journal of Educational Research, 93(2019), 3352. Retrieved from https://doi.org/10.1016/j.ijer.2018.10.001.

Schaeffer, M. W., Rozek, C. S., Berkowitz, T., Levine, S. C. and Beilock, S. L. (2018). Disassociating the relation between parents' math anxiety and children's math achievement: Long-term effects of a math app intervention. Journal of Experimental Psychology: General, 147(12), 1782-1790. Retrieved from http://dx.doi.org/10.1037/xge0000490.

Shute, V. J, Hansen, E. G., Underwood, J. S. and Razzouk, R. (2011). A Review of the Relationship between Parental Involvement and Secondary School Students' Academic Achievement. Education Research International, 2011, 1-10. Retrieved from https://doi.org/10.1155/2011/915326.

Silinskas, G., Kiuru, N., Aunola, K., Lerkkanen, M. K. and Nurmi, J. E. (2015). The developmental dynamics of children's academic performance and mothers' homework-related affect and practices. Developmental Psychology, 51(4), 419-433. Retrieved from http://dx.doi.org/10.1037/a0038908.

Silva, A. y Tabernero, C. (2013). Escuela y familia: Alianza estratégica para la prevención del maltrato infantil. Revista EDUCARE, 17(2). Recuperado de http://revistas.upel.edu.ve/index.php/educare/article/view/1111.

Stevenson, D. L. and Baker, D. P. (1987). The family-school relation and the child's school performance. Child Development, 58(5), 1348-1357. Retrieved from http://dx.doi.org/10.2307/1130626.

Sui-Chu, E. and Willms, J. D. (1996). Effects of Parental Involvement on Eighth-Grade Achievement. Sociology of Education, 69(2), 126-141. Retrieved from https://doi.org/10.2307/2112802.

Tan, T. X., Kim, E. S., Baggerly, J., Mahoney, E. E. and Rice, J. (2017). Beyond adoption status: Post-adoptive parental involvement and children's reading and math performance from kindergarten to first grade. American Journal of Orthopsychiatry, 87(3), 337-346. Retrieved from http://dx.doi.org/10.1037/ort0000216. 


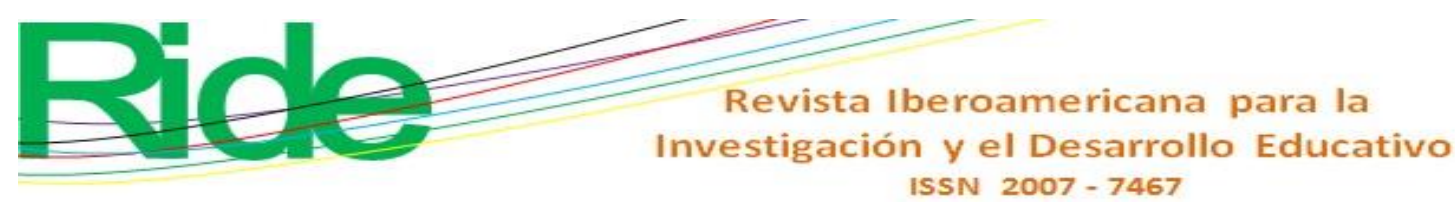

Tardif-Grenier, K. and Archambault, I. (2016). Validation du Questionnaire sur l'implication parentale dans le suivi scolaire (QIPSS) chez des parents d'élèves du primaire en contexte défavorisé et pluriethnique. Revue Européenne de Psychologie Appliquée, 66(3), 139-150. Retrieved from https://doi.org/10.1016/j.erap.2016.04.007.

Tazouti, Y. and Jarlégan, A. (2016). The mediating effects of parental self-efficacy and parental involvement on the link between family socioeconomic status and children's academic achievement. Journal of Family Studies. Retrieved from https://doi.org/10.1080/13229400.2016.1241185.

Ucus, S., Garcia, A., Esteraich, J. and Raikes, H. (2017). Predictors and behavioural outcomes of parental involvement among low-income families in elementary schools, United States. Early Child Development and Care. Retrieved from https://doi.org/10.1080/03004430.2017.1385609.

Valdemoros, Á., Ponce de León, A., Ramos, R. y Sanz, E. (2011). Pedagogía de la convivencia y educación no formal: un estudio desde el ocio físico-deportivo, los valores y la familia. European journal of education and psychology, 4(1), 33-49. Recuperado de https://formacionasunivep.com/ejep/index.php/journal/article/view/65/90. 


\begin{tabular}{|c|c|}
\hline 1) Rol de Contribución & 2) Autor (es) \\
\hline Conceptualización & Edwin Alexis Mayorquin Reyes (igual) / Aníbal Zaldívar Colado (igual) \\
\hline Metodología & Aníbal Zaldívar Colado \\
\hline Software & No Aplica. \\
\hline Validación & Edwin Alexis Mayorquin Reyes \\
\hline Análisis Formal & Edwin Alexis Mayorquin Reyes (igual) / Aníbal Zaldívar Colado (igual) \\
\hline Investigación & Edwin Alexis Mayorquin Reyes (igual) / Aníbal Zaldívar Colado (igual) \\
\hline Recursos & Edwin Alexis Mayorquin Reyes (igual) / Aníbal Zaldívar Colado (igual) \\
\hline Curación de datos & Edwin Alexis Mayorquin Reyes (igual) / Aníbal Zaldívar Colado (igual) \\
\hline $\begin{array}{l}\text { Escritura - Preparación del } \\
\text { borrador original }\end{array}$ & Edwin Alexis Mayorquin Reyes (igual) / Aníbal Zaldívar Colado (igual) \\
\hline Escritura - Revisión y edición & Edwin Alexis Mayorquin Reyes (igual) / Aníbal Zaldívar Colado (igual) \\
\hline Visualización & Edwin Alexis Mayorquin Reyes \\
\hline Supervisión & Edwin Alexis Mayorquin Reyes (igual) / Aníbal Zaldívar Colado (igual) \\
\hline Administración de Proyectos & Edwin Alexis Mayorquin Reyes (igual) / Aníbal Zaldívar Colado (igual) \\
\hline Adquisición de fondos & Aníbal Zaldívar Colado \\
\hline
\end{tabular}

